\title{
Causes of Death After Kidney Transplantation
}

\author{
Graeme F. Washer, MB, ChB, FRACS, Gerhard P. J. Schröter, MD, Thomas E. StarzI, MD, \\ PhD, and Richard Weil III, MD \\ Departments of Surgery, the University of Colorado Health Sciences Center, and the Denver \\ Veterans Administration Hospital, Denver
}

\begin{abstract}
During the five-year period September 1974 through August 1979, two hundred nine consecutive patients received their first kidney transplant in Denver. During 2.5 to 7.5 years of follow-up, 54 patients (26\%) died. Infection was the leading cause of death during all intervals and was responsible for $22(41 \%)$ of the 54 deaths. Pneumonia was primarily responsible for 14 of the 22 deaths from infection. The other causes of death were cardiovascular problems in 11 patients (20\%), suicide in eight patients (15\%), gastrointestinal (GI) tract problems in seven patients (13\%), malignant neoplasms in two patients $(4 \%)$, and miscellaneous problems in four patients (7\%). Twenty-six (48\%) of the 54 deaths occurred more than one year after primary transplantation; 12 of these 26 patients had already returned to chronic hemodialysis. To minimize mortality after transplantation, patients and their physicians must remain alert to the ongoing risks to which these patients are exposed, including the risks of sudden death from infection, myocardial infarction, pulmonary embolus, suicide, or GI tract perforation.
\end{abstract}

In the many reports of kidney transplantation published during the past 20 years, which encompass early developmental work as well as more recent refinements, infection has almost invariably been identified as the chief cause of death after transplantation. ${ }^{1-3}$ Although the relationship between immunosuppression, particularly excessive immunosuppression, and mortality from sepsis was recognized at the outset, ${ }^{4}$ it has not yet been feasible to eliminate deaths from infection entirely, because of a continuing lack of methods for accurately providing optimal immunosuppression to each kidney transplant patient.

A new analysis of the causes of death after kidney transplantation seems timely because of a growing tendency for the follow-up of kidney transplant patients to be carried out not at transplant centers, but by general physicians and nephrologists, whose experience in the care of transplant patients may be limited. It is hoped that this new analysis of mortality will provide information useful to the many physicians outside transplant centers who are now caring for kidney transplant patients.

\section{METHODS}

We have reviewed the results of 209 consecutive patients who received their first kidney transplant at the University of Colorado Health Sciences Center and the Denver Veterans Administration Hospital during the five-year period September 1974 through August 1979. By means of inpatient chart review, outpatient chart review, and telephone calls to patients and families, follow-up information for all patients has been updated to Feb 1, 1982. The

Reprint requests to Box C-304, University of Colorado Health Sciences Center, 4200 E Ninth Ave. Denver. CO 80262 (Dr Weil). 
range of follow-up for the 155 surviving patients is 2.5 to 7.5 years (mean, 58 months). Patients who received a first kidney transplant before September 1974 were excluded, although some of these patients underwent retransplantation during the study period. Two patients who received simultaneous kidney and pancreas transplants were also excluded from analysis.

The mean age of the patients at the time of primary transplantation was 34.4 years (range, 4.0 to 63.0 years). Twenty-two patients (11\%) had diabetes.

Of the 209 first grafts in the 209 study patients, 128 grafts were from cadaver donors and 81 grafts were from living related donors. These 209 patients had a total of 265 transplants during the study period; 56 were retransplants, of which 53 were from cadaver donors and three were from living related donors. The 56 retransplants were carried out in 44 patients; 36 patients had second transplants and eight patients had three or more transplants.

All of the patients received azathioprine and prednisone. One hundred twenty-eight patients received either antilymphocyte globulin produced in Denver or antithymocyte globulin. From February 1978 through August 1979, 67 patients had thoracic duct drainage. ${ }^{5}$ Cyclosporin A was used in Denver beginning in December $1979^{6}$; four patients in the study group received this agent for retransplantation. Blood transfusions were given only for anemia, and not as immunologic preparation. The HLA matches were variable in the related transplant patients and were generally poor (zero to one matched antigens) in the recipients of cadaver grafts; DR (HLA-D-locus-related) typing was not done. Six patients were given transplants despite strongly positive direct cytotoxic cross-matches against their cadaver donors, after preparation by thoracic duct drainage, in an effort to develop a method for successfully performing transplants in highly sensitized patients; the other 203 patients had negative cytotoxic crossmatches against their donors. The details of managment have been described previously. ${ }^{7}$

Splenectomy was carried out in 101 patients (48.3\%), almost always before transplantation, because of hypersplenism manifested by low peripheral WBC count. In some patients, the splenectomy was done at the time of bilateral nephrectomy for pyelonephritis, severe nonfluid-dependent hypertension, vesicoureteral reflux, or infected polycystic kidneys.

\section{RESULTS}

During the 2.5 to 7.5 years of follow-up, 54 (26\%) of the 209 patients died. In 30 (56\%) of the 54 deaths, autopsy reports were available for the purpose of establishing the cause of death; in the remaining 24 deaths, cause of death was determined on clinical grounds. The mortality rate for related kidney recipients was 43 of $128(34 \%)$. The mortality rate for patients who received a primary graft and at least one retransplant during the study period was 12 of $44(27 \%)$. The mortality rate for diabetic patients was 11 of $22(50 \%)$. The mortality rate for splenectomized patients was 24 of $101(24 \%) ; 77$ of $155(50 \%)$ of the survivors had undergone splenectomy and 24 of 54 (44\%) of the patients who died had undergone splenectomy.

Graft function, as of 1982 for survivors and just before terminal events for patients who died, is outlined in Table 1. As of February 1982, 46 of 155 (30\%) of the survivors were again undergoing dialysis; 101 of $155(65 \%)$ of the survivors had functioning grafts with serum creatinine levels of $2.0 \mathrm{mg} / \mathrm{dL}$ or less; 8 of $155(5 \%)$ had serum creatinine levels greater than $2.0 \mathrm{mg} / \mathrm{dL}$. Among the patients who died, the grafts had already failed in 19 (35\%) of the 54 patients; these patients had returned to chronic hemodialysis before the onset of their terminal illness or terminal event. Twenty-two (41\%) of the patients who died 
had serum creatinine levels of $2.0 \mathrm{mg} / \mathrm{dL}$ or less, and 13 (24\%) had functioning grafts, with serum creatinine levels greater than $2.0 \mathrm{mg} / \mathrm{dL}$.

The causes of death and the time intervals between first transplantation and death are listed in Table 2. Death occurred from nine days to 59 months after first transplantation. The causes of death were infection in 22 of 54 patients $(41 \%)$, cardiovascular disease in 11 of 54 patients (20\%), suicide in 8 of 54 patients (15\%), gastrointestinal (GI) tract problems in 7 of 54 patients (13\%), malignant neoplasms in 2 of 54 patients (4\%), and miscellaneous causes in 4 of 54 patients (7\%). Eighteen (33\%) of the 54 deaths occurred within the first three months after primary transplantation; ten (19\%) of the 54 deaths occurred between four and 12 months after transplantation; and $26(48 \%)$ of the 54 deaths occurred more than one year after the primary transplant. The Figure illustrates actuarial patient survival for all 209 patients, as well as the subgroups of 128 cadaver-graft recipients and 81 related-graft recipients. The actuarial results were calculated using the method of Merrill and Schulman. ${ }^{8}$ The period of greatest risk of death was the first six months after transplantation; but even after this interval, there was a continuing threat to life.

\section{Death From Infection}

Overwhelming infection was the primary cause of death in 22 patients, of whom five were diabetic. Death occurred nine to 59 months after transplant (mean, 13.6 months), and 16 of these had functioning transplants at the time of death.

The most common clinical picture of sepsis, occurring nine days to 14 months after surgery (mean, six months) in 11 patients was refractory pneumonia with respiratory failure. Eight of these patients had functioning transplants (six, first; two, second), and six patients had serum creatinine levels less than $2.0 \mathrm{mg} / \mathrm{dL}$. In four patients, it was not possible to identify the dominant organism. In others, a variety of organisms was isolated, including Pneumocystis carinni (four patients), cytomegalovirus (CMV) (three patients), Aspergillus (one patient), Cryptococcus (one patient), and Nocardia (one patient). In addition, two patients had systemic sepsis caused by Staphylococcus plus Pseudomonas and Staphylococcus plus Klebsiella.

Fatal pneumococcal septicemia occurred in three patients at 12, 22, and 59 months (mean, 31 months), all of whom had previously undergone splenectomy. One of these patients, a child who received a transplant for oxalosis and had otitis media develop, has been described elsewhere. ${ }^{9}$ All three had functioning transplants, and two had excellent long-term function of their primary graft; all three were in good stable condition until hours or a few days before death.

Four diabetic patients had sepsis from soft-tissue infections; three of these arose in the extremities five, ten, and 20 months after transplant. The fourth patient died of overwhelming Serratia sepsis 24 days after transplant. Three of the four patients had returned to dialysis before the onset of their terminal illness. One patient had good graft function at the time of death.

Four other nondiabetic patients died of sepsis caused by staphylococcal bacterial endocarditis, intraabdominal abscess of uncertain cause, enterobacterial septicemia, and infected arteriovenous fistula (one patient each).

Infection was a contributory but not primary cause of death in another nine patients. Five patients had pneumonia with single or multiple organisms (Pneumocystis, CMV, Nocardia, Aspergillus, unknown); four had peritoneal sepsis with or without systemic infection (Staphylococcus, Candida, Klebsiella) in association with GI tract perforations. 


\section{Death From Cardiovascular Causes}

Five patients (mean age at transplant, 47 years; range, 40 to 58 years) died of myocardial infarction at 1,2,13, 25, and 32 months after surgery. Three more patients died of pulmonary emboli. (One other patient had an undetected myocardial infarction discovered incidentally at autopsy, and pulmonary emboli were seen in the lungs of several additional patients who underwent autopsy.)

One diabetic patient suffered a cardiac arrest on dialysis 46 months after transplant, and one severely hypertensive patient with satisfactory renal function 32 months after transplant was dead on arrival at the hospital and was presumed to have had a myocardial infarction. One patient died of congestive heart failure while receiving dialysis at another center 23 months after transplant.

All 11 patients dying of cardiovascular causes were at least 40 years of age (mean, 49 years; range, 40 to 60 years) at the time of transplantation. Four of these patients had returned to dialysis, and only four had serum creatinine levels of $2.0 \mathrm{mg} / \mathrm{dL}$ or less. Three were diabetic.

\section{Death From Suicide}

Eight younger patients (mean age at transplant, 27.8 years; range, 17 to 38 years) died as a result of overt or veiled suicide, including refusal to undergo dialysis after unsuccessful transplantation. Most had recognizable psychological instability before transplantation and all but one had rejected one or more transplants. One 26-year-old woman who appeared to have been rehabilitated and who had perfect renal function after primary HLA-identicalrelated transplantation shot herself five months after surgery. The remaining seven patients survived from one to 29 months after graft failure and return to dialysis. Only one patient had a protracted hospital course with multiple retransplants. Two of the patients who died of suicide were diabetic.

\section{Death From GI Tract Causes}

Seven patients died of GI tract disorders; most of these deaths were complicated by sepsis. The mean age at the time of transplantation for this group was 36.5 years (range, 35 to 50 years), and none of the patients were diabetic.

Perforations of the GI tract from duodenal ulcer, gastric ulcer, small bowel, and sigmoid colon, were each responsible for the death of one patient. These four patients died of peritonitis, with or without abscess formation, despite early diagnosis and aggressive surgical management. Additional contributing causes were Nocardia pneumonia and pulmonary emboli in one patient and Klebsiella septicemia in another patient.

Severe acute pancreatitis one month after transplantation was responsible for the death of a 35-year-old woman; a 35-year-old man died 42 months after transplantation from rupture of a pancreatic abscess.

One 47-year-old man with a history of ulcer disease manifested duodenal ulceration one month after transplantation, and, despite truncal vagotomy and antrectomy after an initial hemorrhage, exsanguinated one week later. His course was complicated by a combined Aspergillus and CMV pneumonia, and a silent myocardial infarction was found at autopsy.

\section{Death From Malignant Neoplasms}

Two patients, 48 and 50 years old at the time of transplantation, died of malignant neoplasms - one of undifferentiated sarcoma of the bone marrow (which could not be more precisely characterized), 19 months after transplant, and one of diffuse lymphoma 
originating in deep cervical lymph nodes, 40 months after transplant. Both patients had functioning first grafts and had not received unusual amounts of immunosuppressive drugs, although the former patient was treated for a mild rejection episode just before the diagnosis of a malignant neoplasm.

\section{Death From Miscellaneous Causes}

There were four deaths from other causes; three occurred within the first three months after surgery and were related to technical complications. A 20-year-old man died of aspiration 13 days after transplant after anesthesia for evacuation of a small peritransplant hematoma. A 53-year-old man with a severe pneumonia, the cause of which was never determined, died of hemorrhage after transbronchial lung biopsy. Both had functioning first transplants. A 22year-old man with CMV pneumonia and hepatitis died of hemorrhage after a percutaneous liver biopsy three months after transplant, with excellent renal function. One diabetic patient, who had poorly controlled diabetes on dialysis after a rejected transplant, died after a hypoglycemic seizure at another hospital 29 months after transplantation.

\section{COMMENT}

More than 15 years ago, in March 1967, one of us (T.E.S.) wrote an editorial entitled "Death After Transplantation: An Analysis of 60 Cases." 10 In this report of the 1962-1965 Denver experience, the leading causes of 16 early deaths were pulmonary embolus $(5 / 16)$ and sepsis (4/16); the leading cause of 44 subsequent deaths was infection (38/44). During the more than 15-year interval since the editorial was published, infection has continued to be the main cause of mortality after kidney transplantation; but a general decrease in the amount of corticosteroid immunosuppression has reduced the mortality rate. ${ }^{11}$ The 49 of 107 (46\%) mortality rate from the 1962-1965 Denver series of kidney allografts, with mean follow-up of two years, has been reduced in the 1974-1979 Denver series to 26\%, with mean followup of five years. This reduction in mortality is consistent with the experience in other major centers. ${ }^{12,13}$ Nevertheless, chronic immunosuppression in kidney transplant patients with previous chronic renal failure, who sometimes have other systemic illnesses, eg, diabetes or systemic vasculitis, continues to be associated with ongoing morbidity and mortality.

The causes of death in kidney transplant patients consist of multiple disease processes involving multiple-organ systems. The kidney transplant patient is subject, for example, to infection, ${ }^{14}$ hyperlipidemia, ${ }^{15}$ myocardial infarction, ${ }^{16}$ cerebrovascular accident, ${ }^{16}$ psychiatric difficulties, ${ }^{17}$ GI tract complications, including peptic ulcer disease with gastroduodenal bleeding or perforation, ${ }^{18,19}$ pancreatitis, ${ }^{20}$ hepatitis and liver failure, ${ }^{21}$ colon perforation, ${ }^{22}$ and cancer. ${ }^{23}$

In the early years, technical accidents were responsible for a number of perioperative deaths. ${ }^{24}$ Operative mortality has now become distinctly uncommon. In the 1974-1979 Denver experience, there were no deaths directly attributable to the transplant operation itself, although one patient died of anesthetic complications following evacuation of a wound hematoma. There have been no urologic complications leading to death in this series.

\section{Infection}

Infection has been the major cause of death in almost all reports of kidney transplantation, ${ }^{1,4,12,13}$ both soon and late after transplantation. Multiple organisms are commonly found, and energetic diagnosis and treatment of all infections, especially pneumonia, ${ }^{25}$ is essential.

Prophylactic antibiotics have not been used in our patients, but meticulous attention to sterile technique at the time of surgery has been practiced throughout the study. Wound 
infection has been uncommon and was not responsible for any deaths in the current study group.

Of our 22 patients who died of infection, 16 had transplant function; ten of these had serum creatinine levels of $2.0 \mathrm{mg} / \mathrm{dL}$ or less, with a mean survival of 6.1 months (range, 0 to 22 months). Two of these had undergone retransplantation, one after immediate failure of the first graft and one after chronic rejection and retransplantation without return to dialysis. The latter patient, a 20-year-old woman who died of fungal pneumonia, died six weeks after retransplantation. Although patients who have had a previously successful transplant often strongly desire retransplantation without interval dialysis, the risk of immediate retransplantation in some chronically immunosuppressed, debilitated patients may be prohibitively high.

It has sometimes been thought ${ }^{10}$ that patients dying of infection have had complicated postoperative courses with multiple rejection episodes and high total doses of immunosuppressive therapy. Eleven of our 22 patients who died of infection had satisfactory stable function of their first graft (mean serum creatinine level, $2.0 \mathrm{mg} / \mathrm{dL}$ or less), and two patients had good function of a second graft; ten of these 13 patients had suffered no rejection episodes of their existing graft before their terminal illness.

Sudden septicemia in patients whose conditions are stable, particularly those who have undergone splenectomy, might have been avoided in the three Denver patients who died of pneumococcal sepsis by previous administration of the currently available polyvalent pneumococcal vaccine; however, immunization against infection by encapsulated organisms has been less successful in these immunosuppressed patients who have undergone splenectomy than in a non-immunosuppressed population of patients who have undergone splenectomy. ${ }^{26}$ In addition, the value of the polyvalent pneumococcal vaccine is limited to the serotypes included in the vaccine. In the Denver patients, the overall mortality in patients who had undergone splenectomy (24\%) was not notably different from the mortality of the entire group of patients (26\%). In recent years, since the end of the current study period, polyvalent pneumococcal vaccine has been administered prospectively to patients undergoing splenectomy to try to provide protection against the most common types of pneumococcal infection.

In any patient with life-threatening infection, the source of the infection must be eradicated to the maximum extent feasible, which may require early operation; appropriate antimicrobial therapy must be initiated early, and immunosuppression must be promptly lowered to a safe level.

\section{Cardiovascular Deaths}

Cardiovascular deaths continue to constitute an important cause of mortality; however, such deaths are particularly difficult to avoid when older, higher-risk patients are given transplants. ${ }^{3}$ Three of our eleven patients who died of cardiovascular causes were diabetic. In the future, it may be feasible to prevent or reverse diabetic vascular disease by an implantable insulin pump or a pancreas transplant, but these approaches are still experimental. The average age of the group of patients who died of cardiovascular causes (49 years) was higher than that of the groups who died of other causes, except for the two patients who died of malignant neoplasms (Table 2). As new pharmacologic methods of managing plasma lipid disorders become available, it may become possible to prevent or even reverse the arterial pathologic conditions ${ }^{29}$ and hyperlipidemia ${ }^{15}$ that are so destructive to some of these patients. Early coronary artery bypass may also increase longevity for some patients, ${ }^{30}$ although the indications for this procedure are still controversial.

Thrombophlebitis and pulmonary emboli must be diagnosed and treated without delay. 


\section{Suicide}

The psychological trauma and depression associated with end-stage renal disease and transplantation may be devastating. ${ }^{17}$ Energetic situational and psychiatric support is almost always important. Many patients receiving chronic dialysis have successful transplantation as their single remaining goal; it is therefore not surprising that the despair that may accompany graft failure and the alteration of physical appearance by steroid treatment, particularly among younger patients, can lead to suicide.

Psychological disturbances induced by high-dose prednisone in the early postoperative period are also a concern in some patients, although the majority of deaths by suicide have been seen more than a year after transplantation.

Five of our eight patients who committed suicide left the hospital with a functioning graft but enjoyed an average of only 5.6 months of satisfactory renal function before returning to dialysis or before they died. More aggressive psychiatric treatment might have prevented some of these tragic deaths.

\section{GI Tract}

Some immunosuppressed patients with acute peritonitis have minimal symptoms; nevertheless, prompt evaluation of any new abdominal symptoms may result in early diagnosis and early treatment of life-threatening peritonitis from GI tract perforations. ${ }^{22}$

Death from bleeding peptic ulcers occurred in one patient. In the past, ulcer surgery (highly selective vagotomy with pyloroplasty) was recommended for patients with a history of ulceration; more recently, cimetidine therapy has to some extent supplanted vagotomy, although nephrotoxicity has been reported. ${ }^{27}$

In the 1974-1979 Denver patients, we have not found chronic liver failure to be a major cause of death, in contrast to the experience at other centers, including the Peter Bent Brigham Hospital in Boston. ${ }^{28}$ Although the follow-up period in this Denver series is shorter than in the Boston report, it is not clear why death from chronic liver failure was not observed in Denver. Chronic mild hepatocellular dysfunction or chronic hepatitis antigenemia have not been regarded as contraindications to transplantation in Denver.

Transplant patients or candidates for transplantation must be carefully screened and treated early for ulcerative GI tract disease, in which perforation may be fatal. Peptic ulcer disease that is refractory to cimetidine and antacid therapy may require operative treatment. Symptomatic diverticulosis coli may require colon resection. ${ }^{22}$ Patients with a history of pancreatitis must abstain from alcohol and should have periodic ultrasound examinations of the pancreas.

\section{Malignant Neoplasms}

Malignant neoplasms associated with immunosuppressive therapy are a serious long-term danger associated with organ transplantation, and the Denver registry reports have comprehensively detailed the spectrum and incidence of various tumors. ${ }^{23}$

Induction of specific immune tolerance to the homografted organ without broad immunosuppression, which is the goal of antirejection treatment, has yet to be realized. Preliminary experience with cyclosporin A as a steroid-sparing immunosuppressive ${ }^{6}$ suggests that this potent immunosuppressive agent, compared with earlier immunosuppressive agents, is not associated with a lowered risk of malignancy. Polyclonal lymphoproliferative disorders have recently been reported in immunosuppressed transplant patients $^{31}$; and there is a report of transformation from a polyclonal lymphoproliferative 
disorder into a monoclonal malignant lymphoma. ${ }^{32}$ Continuous surveillance and early biopsy of any suspicious cutaneous or other tumor is essential in all transplant patients. Malignant neoplasms may be difficult to control in an immunosuppressed patient; if there is a possibility of cure, management should include prompt discontinuation of immunosuppressive therapy and aggressive treatment of the cancer. In patients with advanced malignant neoplasms with little probability of cure, it is occasionally kinder to continue immunosuppression to maintain the benefit of kidney function during the end of the patient's life.

\section{Miscellaneous}

The four deaths listed as "miscellaneous" were all at least potentially avoidable: aspiration immediately following general inhalation anesthesia and extubation; intrabronchial hemorrhage after transbronchial lung biopsy; intra-abdominal hemorrhage following percutaneous liver biopsy in CMV hepatitis; and hypoglycemic seizure in a diabetic patient who had returned to chronic hemodialysis. The other ten deaths in our 22 diabetic patients, who had a mortality rate of 11 of $22(50 \%)$, were caused by infection in five patients, myocardial infarction in two patients, pulmonary embolus in one patient, and suicide in two patients. The problems of infection and cardiovascular disease in diabetic patients have been well defined ${ }^{33}$ and are perhaps being better managed in recent years ${ }^{34}$ since the end of the current study.

\section{References}

1. Tapia HR, Holtey KE, Woods JE, et al. Causes of death after renal transplantation. Arch Intern Med. 1973; 131:204-209. [PubMed: 4566574]

2. Tilney NL, Strom TB, Vineyard GC, et al. Factors contributing to the declining mortality rate in renal transplantation. N Engl J Med. 1978; 299:1321-1325. [PubMed: 101845]

3. Salvatierra O Jr, Potter D, Cochrum KC, et al. Improved patient survival in renal transplantation. Surgery. 1976; 79:166-171. [PubMed: 1108257]

4. Starzl, TE. Experience in Renal Transplantation. Philadelphia: WB Saunders Co; 1964. p. 213-238.

5. Starzl TE, Klintmalm GBG, Iwatsuki S, et al. Late follow-up after thoracic duct drainage in cadaveric renal transplantation. Surg Gynecol Obstet. 1981; 153:377-382. [PubMed: 7022708]

6. Starzl TE, Klintmalm GBG, Weil R III, et al. Cyclosporin A and steroid therapy in 66 cadaver kidney recipients. Surg Gynecol Obstet. 1981; 153:486-494. [PubMed: 6269238]

7. Weil R III, Schröter GPJ, West JC, et al. A 14-year experience with kidney transplantation. World J Surg. 1977; 1:145-156. [PubMed: 141173]

8. Merrill M, Shulman LE. Determination of prognosis in chronic disease illustrated by systemic lupus erythematosus. J Chronic Dis. 1955; 1:12-32. [PubMed: 13233308]

9. Solomons, CC.; Schröter, GPJ.; Guggenheim, SJ., et al. Pediatric kidney transplantation for congenital oxalosis. In: Strauss, J., editor. Pediatric Nephrology: Renal Failure. Vol. 4. New York: Garland STPM Press; 1978. p. 217-229.

10. Hill RB Jr, Dahrling BE II, Starzl TE, et al. Death after transplantation. Am J Med. 1967; 42:327333. [PubMed: 4381194]

11. Strom TB. The improving utility of renal transplantation in the management of end-stage renal disease. Am J Med. 1982; 73:105-124. [PubMed: 7046437]

12. Murray JE, Tilney NL, Wilson RE. Renal transplantation: A 25 year experience. Ann Surg. 1976; 184:565-573. [PubMed: 791162]

13. Matas AJ, Simmons RL, Buselmeier TJ, et al. The fate of patients surviving three years after renal transplantation. Surgery. 1976; 80:390-395.

14. Murphy JF, McDonald FD, Dawson M, et al. Factors affecting the frequency of infection in renal transplant recipients. Arch Intern Med. 1976; 136:670-677. [PubMed: 776110]

15. Ibels LS, Alfrey AC, Weil R III. Hyperlipidemia in adult, pediatric and diabetic renal transplant recipients. Am J Med. 1978; 64:634-642. [PubMed: 206137] 
16. Advisory Committee to the Renal Transplant Registry: The 13th report of the Human Renal Transplant Registry. Transplant Proc. 1977; 9:9-26.

17. Kemph, JP. Psychotherapy with donors and recipients of kidney transplants. In: CastelnouvaTedesco, P., editor. Psychiatric Aspects of Organ Transplantation. New York: Grune \& Stratton Inc; 1971. p. 144-158.

18. Owens ML, Passuro E, Wilson SE, et al. Treatment of peptic ulcer disease in the renal transplant patient. Ann Surg. 1977; 186:17-21. [PubMed: 327953]

19. Spanos PK, Simmons RL, Rattazzi LC, et al. Peptic ulcer disease in the transplant recipient. Arch Surg. 1974; 109:193-197. [PubMed: 4602992]

20. Fernandez JA, Rosenberg JC. Post-transplantation pancreatitis. Surg Gynecol Obstet. 1976; 143:795-798. [PubMed: 790614]

21. Berne TV, Chatterjee SN, Craig JR, et al. Hepatic dysfunction in recipients of renal allografts. Surg Gynecol Obstet. 1975; 141:171-175. [PubMed: 168654]

22. Carson SD, Krom RAF, Uchida K, et al. Colon perforation after kidney transplantation. Ann Surg. 1978; 188:109-113. [PubMed: 352278]

23. Penn I. Tumor incidence in human allograft recipients. Transplant Proc. 1979; 11:1047-1051. [PubMed: 377608]

24. Weil R III, Simmons RL, Tallent MB, et al. Prevention of urological complications after kidney transplantation. Ann Surg. 1971; 174:154-160. [PubMed: 4933525]

25. Fanta $\mathrm{CH}$, Pennington JE. Fever and new lung infiltrates in the immunocompromised host. Clin Chest Med. 1981; 2:19-39. [PubMed: 7030595]

26. Hosea SW, Burch CG, Brown EJ, et al. Impaired immune response of splenectomized patients to polyvalent pneumococcal vaccine. Lancet. 1981; 1:804-807. [PubMed: 6111673]

27. Rudnick MR, Bastl CP, Elfenbein IB, et al. Cimetidine-induced acute renal failure. Ann Intern Med. 1982; 96:180-182. [PubMed: 7059066]

28. Kirkman, RL.; Strom, TB.; Weir, MR., et al. Late mortality and morbidity in recipients of longterm renal allografts, abstracts of the American Society of Transplant Surgeons Annual Meeting; Chicago. June 2-3,1982; p. 48-49.

29. Ibels LS, Alfrey AC, Huffer WE, et al. Arterial calcification and pathology in uremic patients undergoing dialysis. Am J Med. 1979; 66:790-796. [PubMed: 443254]

30. Braun WE, Phillips D, Vidt DG, et al. Coronary arteriography and coronary artery disease in 99 diabetic and nondiabetic patients on chronic hemodialysis or renal transplantation programs. Transplant Proc. 1981; 13:128-135. [PubMed: 7022809]

31. Hanto DW, Sakamoto K, Purtilo DT, et al. The Epstein-Barr virus in the pathogenesis of posttransplant lymphoproliferative disorders: Clinical, pathologic and virologic correlation. Surgery. 1981; 90:204-213. [PubMed: 6266059]

32. Hanto DW, Frizzera G, Gajl-Peczalska KJ, et al. Epstein-Barr virus-induced B-cell lymphoma after renal transplantation. N Engl J Med. 1982; 306:913-918. [PubMed: 6278307]

33. Najarian JS, Sutherland DER, Simmons RL, et al. Kidney transplantation for the uremic diabetic patient. Surg Gynecol Obstet. 1977; 144:682-690. [PubMed: 191945]

34. Peters C, Sutherland DER, Simmons RL, et al. Patient and graft survival in amputated $v$ nonamputated diabetic primary renal allograft recipients. Transplantation. 1981; 32:498-503. [PubMed: 7041351] 


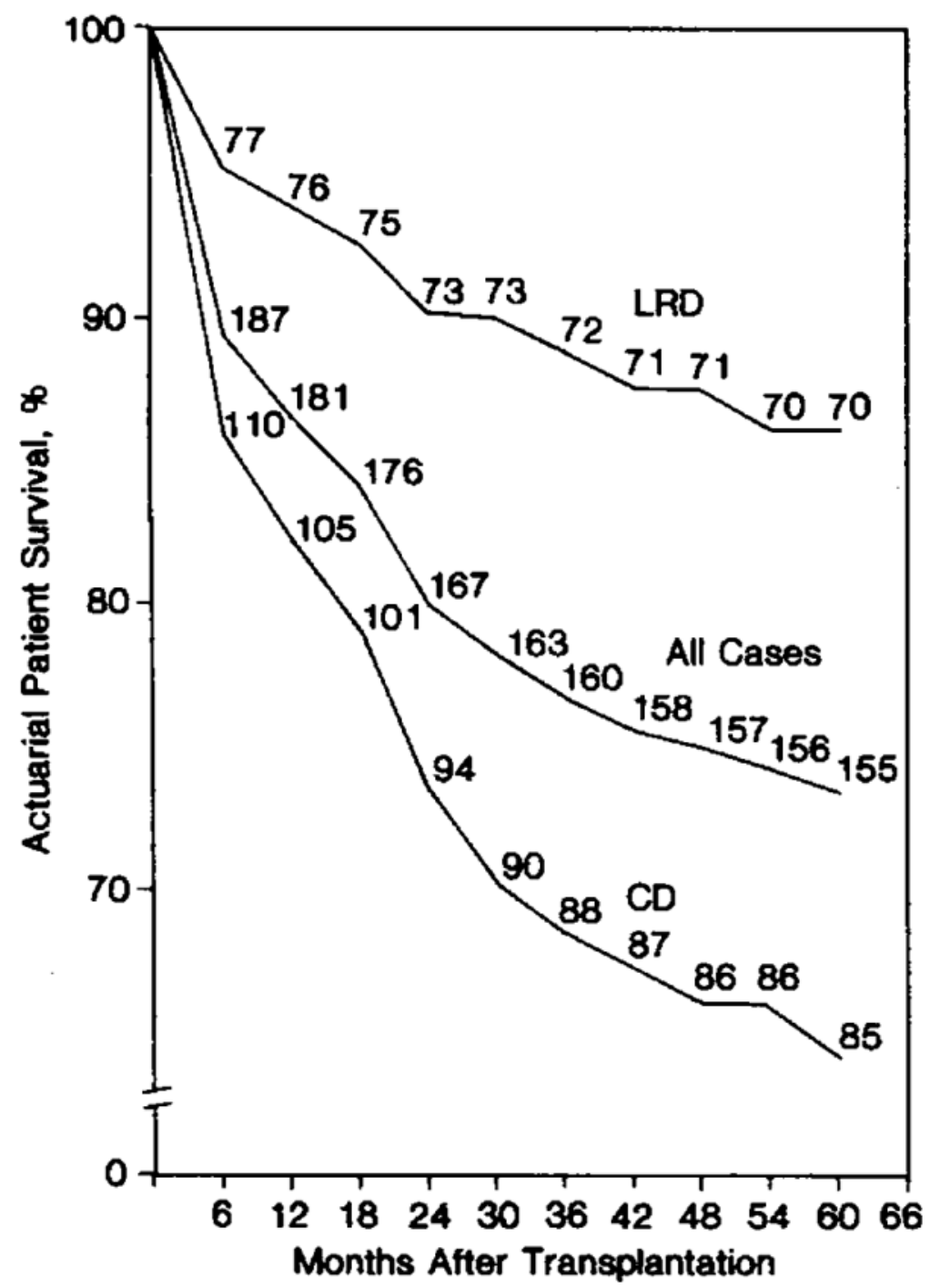

Fig 1.

Actuarial patient survival for 209 patients who received kidney transplants between September 1974 and August 1979. Five-year actuarial patient survival was $86 \%$ for recipients of living-related donor (LRD) kidneys $(\mathrm{N}=81)$ and $64 \%$ for recipients of cadaver donor (CD) kidneys $(\mathrm{N}=128)$. Overall five-year actuarial patient survival was $74 \%$. 
Table 1

Graft Function

\begin{tabular}{|c|c|c|}
\hline & No. $(\%)$ of Survivors $*(N=155)$ & No. $(\%)$ of Deaths $(\mathrm{N}=54)$ \\
\hline \multicolumn{3}{|c|}{ Serum creatinine level, mg/dL } \\
\hline$\leq 2.0$ & $101(65)$ & $22(41)$ \\
\hline$>2.0$ & $8(5)$ & $13(24)$ \\
\hline Dialysis & $46(30)$ & $19(35)$ \\
\hline
\end{tabular}

As of February 1982. 


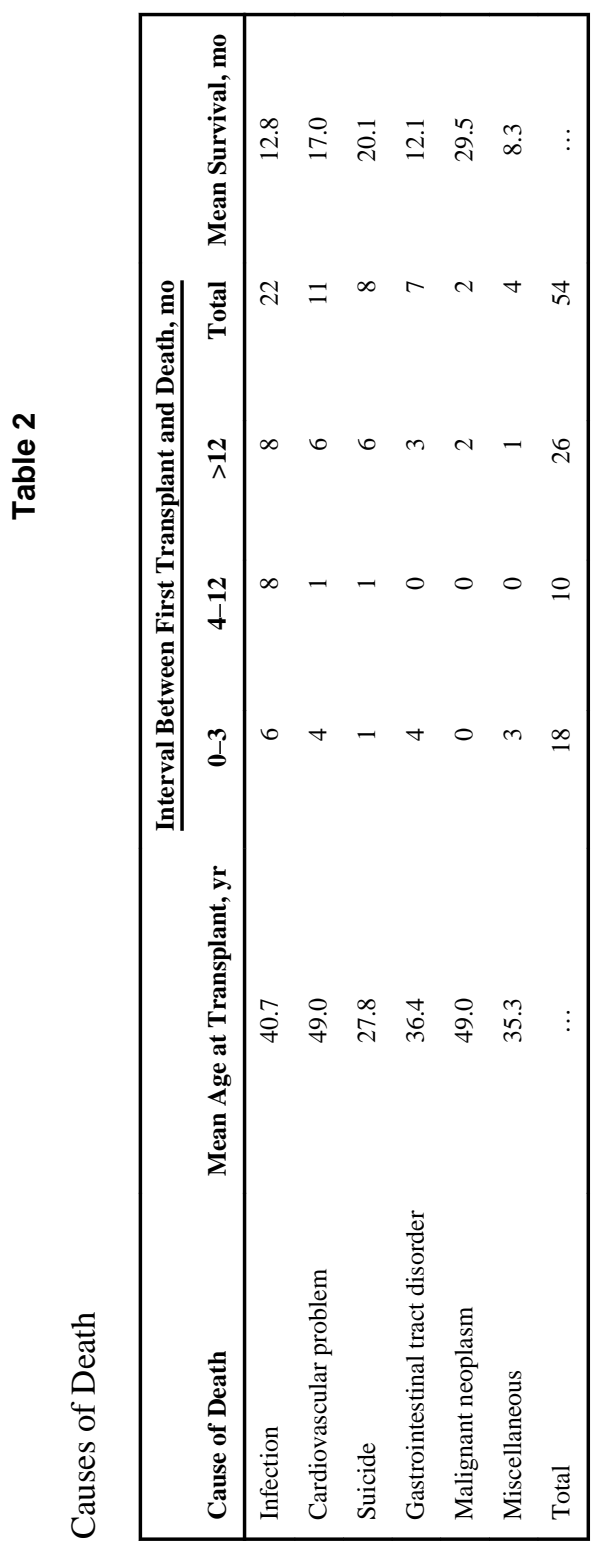

JAMA. Author manuscript; available in PMC 2011 May 10. 